\title{
Dust-acoustic shocks in strongly coupled dusty plasmas
}

\author{
S. E. Cousens, ${ }^{1, *}$ V. V. Yaroshenko, ${ }^{2}$ S. Sultana,${ }^{3}$ M. A. Hellberg,${ }^{4}$ F. Verheest,${ }^{5,4}$ and I. Kourakis ${ }^{1, \dagger}$ \\ ${ }^{1}$ Centre for Plasma Physics, Department of Physics and Astronomy, Queen's University Belfast, BT7 INN Northern Ireland, United Kingdom \\ ${ }^{2}$ Deutsches GeoForschungsZentrum, GFZ, 14473 Potsdam, Germany \\ ${ }^{3}$ Department of Physics, Jahangirnagar University, Dhaka-1342, Bangladesh \\ ${ }^{4}$ School of Chemistry and Physics, University of KwaZulu-Natal, Durban 4000, South Africa \\ ${ }^{5}$ Sterrenkundig Observatorium, Universiteit Gent, Krijgslaan 281, B-9000 Gent, Belgium
}

(Received 18 July 2013; published 4 April 2014)

\begin{abstract}
Electrostatic dust-acoustic shock waves are investigated in a viscous, complex plasma consisting of dust particles, electrons, and ions. The system is modelled using the generalized hydrodynamic equations, with strong coupling between the dust particles being accounted for by employing the effective electrostatic temperature approach. Using a reductive perturbation method, it is demonstrated that this model predicts the existence of weakly nonlinear dust-acoustic shock waves, arising as solutions to Burgers's equation, in which the nonlinear forces are balanced by dissipative forces, in this case, associated with viscosity. The evolution and stability of dust-acoustic shocks is investigated via a series of numerical simulations, which confirms our analytical predictions on the shock characteristics.
\end{abstract}

DOI: 10.1103/PhysRevE.89.043103

PACS number(s): 52.27.Lw, 52.35.Fp, 52.35.Tc, 52.27.Gr

\section{INTRODUCTION}

At small amplitude, waves in plasmas may often be treated as linear phenomena, but as the amplitude of these waves increases, various processes occur which may not be described by linear theory. An important nonlinear effect is that the higher-amplitude parts of a wave travel faster than the lower-amplitude parts, leading to a steepening of the wave front. Wave steepening is not the only mechanism to affect waves in a plasma, and its effects may be mitigated, or even balanced, by other processes. An example of one such effect is dispersion, by which various frequency components in the wave travel at different speeds, causing the wave to be stretched over time. When the effect of wave steepening is balanced by dispersion, wave structures known as solitary waves, or solitons, may be produced. Another process is energy dissipation or damping of the waves in the medium. This involves the transfer of energy from the wave, and a balance between wave steepening and dissipation results in the formation of shock waves. Various techniques have been developed to analytically model nonlinear waves, such as the Sagdeev pseudopotential approach [1] for solitons, and the reductive perturbation method [2], which may be employed whenever the amplitude of the nonlinear wave is small.

A very common type of plasma, and one which is of particular interest to this paper, is a complex or dusty plasma, which consists of massive dust particles in addition to the usual electron and ion components [3]. The dust particles may attain a large net negative charge, since the electrons typically have a larger thermal speed than the ions. Initially, the electrons collide with the dust particles more frequently than the ions. As the dust grains become more negatively charged, electrons are increasingly repelled, reducing the electron flux, while at the same time the ion flux increases, until the equilibrium dust charge is reached when the electron

\footnotetext{
*scousens01@qub.ac.uk

†ioanniskourakissci@gmail.com; www.kourakis.eu
}

and ion fluxes are equal and the charging process is complete. An understanding of dusty plasma physics is necessary to explain various astrophysical phenomena [4] and is important for many industrial and manufacturing applications [5]. The introduction of a massive, charged dust component is found to greatly affect the dynamics of a usual electron-ion plasma. This includes both a modification to existing linear wave modes, and the introduction of new ones, such as the dust-acoustic wave (DAW), in which the pressures of the relatively inertialess electrons and ions provide the restoring force for the inertial dust [6,7]. It is interesting that the large size of the dust particles, as well as their low velocities, allow scientists to view the propagation of dust-acoustic waves in the laboratory in real time [8].

Another interesting aspect of dusty plasma physics is that the dust particles may experience significant electrostatic forces from their neighbors. The coupling parameter $\Gamma=Z_{d}^{2} e^{2} / 4 \pi \epsilon_{0} k_{B} T_{d} n_{d}^{-1 / 3}$ is the ratio between the Coulomb potential energy and the thermal energy of the dust and is used as measure of the relative importance of these electrostatic forces on the motion of the dust particles. Here $Z_{d} e, k_{B} T_{d}$, and $n_{d}$ are the charge, kinetic temperature, and number density of the dust particles, respectively. The high charge number and low kinetic temperature of dust particles often leads to coupling parameters that are greater than unity. In such plasmas the electrostatic forces are of great importance and we class the dust particles as being strongly coupled [9]. When the coupling parameter gets sufficiently large, crystallization can occur, and so dusty plasmas provide an excellent opportunity to study phase transitions, which is another very active topic of research. In a strongly coupled dusty plasma, viscosity often plays an important role in the dynamics of the dust particles and of the properties of the wave modes. Of particular relevance to this study is that the dissipation of energy through viscous forces may provide a balance for nonlinear forces that drive the steepening of the wave front and allow stable dust-acoustic shocks to form.

Gozadinos et al. [10] presented a model for strongly coupled crystalline dusty plasmas, whereby the effects of strong 
coupling are accounted for via an electrostatic "pressure". This model, although originally developed for crystalline plasma structures, has recently been applied as an approximation to the equation of state for strongly coupled plasmas near to the liquid-crystal phase transition. This has included the study of Bohm sheaths [11], double-layer formation [12,13], the linear DAW mode [14], and nonlinear solitary wave structures [15,16]. Yaroshenko et al. [14] found that, by interpreting the strongly coupled interactions as a consequence of an electrostatic "temperature", the transition to a thermal mode at high wave numbers for dispersion curves obtained in previous experimental studies [17] may be accurately predicted.

In Ref. [18] we theoretically investigated weakly nonlinear dust-acoustic solitary waves in a complex plasma in which the dust particles are strongly coupled, with the effects of strong coupling being accounted for by the electrostatic temperature approach. To account for local variations in electrostatic temperature in the vicinity of a wave, we introduced the concept of electrostatic temperature perturbations. In doing so, we noted that including the electrostatic temperature perturbations predicts modifications to the sound speed in the plasma, as well as the amplitude and width of solitary waves.

In this paper, we extend this model to study dust-acoustic shock waves in a dusty plasma in which the dust particles are in a strongly coupled, but still fluid, state. In particular, since we use the electrostatic pressure approach of Gozadinos et al. [10], our results are applicable in the vicinity of the liquid-crystal phase transition. This state is often observed in the laboratory, signified by a coexistence of crystalline structures alongside a viscous fluidlike plasma [19]. A detailed description of the model, along with the key assumptions made, is presented in Sec. II. We use the reductive perturbation method [2], described in Sec. III, to derive both the linear dispersion relation and Burgers's equation for this system in Sec. IV. In addition to this, we then present a discussion on dust-acoustic shocks, as they arise as solutions to the derived Burgers's equation, for the plasma parameters given by Rosenberg et al. [20]. In Sec. V, we present a numerical simulation for dust-acoustic shocks propagating in a plasma system for which they are not a solution to Burgers's equation. This numerical study is based on the parameters presented in Ref. [20] and employs a Runge-Kutta iteration method to integrate Burgers's equation using a specified shock potential profile as an initial condition. Finally, in Sec. VI we discuss the key findings of this approach.

\section{MODEL SYSTEM}

In this section, we present a fluid model for dust-acoustic waves in a one-dimensional, three-component plasma consisting of dust particles, electrons, and ions. This will include a brief discussion on the key assumptions we make in the model and the equations that result from them.

In any particular dusty plasma system the dust particles may have a range of sizes, with charge numbers varying both spatially and temporally. In this paper, we take a simplified case such that the dust particles each have a constant mass $m_{d}$ and a fixed charge number $Z_{d}$. The latter means that we do not include charge exchange processes, which might result in the dust particles gaining or losing charges, in our analysis. This commonly used assumption implies that the study is restricted to plasmas in which the typical (dust-acoustic) time scale differs significantly from (and is, in fact, much larger than) the dust grain charging time. We note, for completeness, that in some situations (where the grain charging time scale is comparable with the excited wave time scale) grain charging processes do have effects on the dynamics of the system [21]. The dust number density, $n_{d}$, is thus conserved, and this is reflected in the continuity equation,

$$
\frac{\partial n_{d}}{\partial t}+\frac{\partial}{\partial x}\left(n_{d} u_{d}\right)=0
$$

where $u_{d}$ is the dust fluid velocity.

We consider three forces on the dust particles. The first is the electrostatic force caused by separation of charges in the plasma. We assume that there are no external electric fields applied to the system, and so this charge separation is caused solely by the plasma being perturbed by a passing wave. We express this force as the gradient of the electrostatic potential $\Phi$.

The second force we consider is due to the mutual electrostatic repulsion of similarly charged dust particles. We model a fluid plasma that is close to the liquid-crystal phase transition, and so to obtain a first approximation for this force, we use the equation of state derived by Gozadinos et al. [10] for dust particles in a crystalline structure. In this model, the coupling energy per particle, $\mathcal{E}_{0}$, is assumed to be of the isotropic screened Coulomb (Debye-Hückel or Yukawa) form between the particle and its nearest neighbors, such that

$$
\mathcal{E}_{0} \simeq N_{n n} \frac{Z_{d}^{2} e^{2}}{4 \pi \epsilon_{0} a} \exp (-\kappa),
$$

where $N_{n n}$ is the number of nearest neighbors, determined by the crystalline plasma structure, and $\kappa=a / \lambda_{D}$ is the normalized interparticle distance, defined as the ratio between the dust interparticle distance $a=n_{d}^{-1 / 3}$ and the Debye screening length in the plasma, $\lambda_{D}=\sqrt{\epsilon_{0} k_{B} T_{i} T_{e} / e^{2}\left(n_{i} T_{e}+n_{e} T_{i}\right)}$. By assuming that the coupling energy is the dominant source of free energy, $F$, in the system such that $F \simeq N_{d} \mathcal{E}_{0}$, where $N_{d}$ is the number of dust particles, Gozadinos et al. [10] determined the pressure via the thermodynamic relation $P_{\star}=$ $-(\partial F / \partial V)_{T_{d}} \simeq n_{d}^{2}\left(\partial \mathcal{E}_{0} / \partial n_{d}\right)_{T_{d}}$. Here $V$ denotes the volume of the plasma, such that $V=N_{d} / n_{d}$. The force exerted on the dust particles is then the gradient of this electrostatic pressure, $\partial P_{\star} / \partial x$. Following the work of Yaroshenko et al. [14], we define the electrostatic temperature via the equation $P_{\star}=$ $n_{d} k_{B} T_{\star}$, which yields

$$
k_{B} T_{\star}=\frac{N_{n n} Z_{d}^{2} e^{2}}{12 \pi \epsilon_{0}} \sqrt[3]{n_{d}}(1+\kappa) \exp (-\kappa) .
$$

For the purposes of this paper, we take $N_{n n}=12$, which is the value for a face-centered cubic lattice. Since we model a fluid plasma, this value of $N_{n n}$ is used only for the sake of analytical tractability, to proceed with numerical computations, and should be taken as an approximation for the system.

We note that we have employed the Debye-Hückel (or Yukawa) model in the derivation of the electrostatic pressure. This is commonly used in the literature $[19,22]$ and has, for 
instance, proven to be useful in providing results that are supported by experiments [23]. For the idealized dusty plasma we describe in this paper, the Debye-Hückel model is therefore used as an approximation to the interaction potential of the dust particles.

Third, we introduce a dissipative force, representing momentum transfer in the system. In principle, energy dissipation in our model is not due to collisions with neutrals but rather is associated with the intrinsic longitudinal viscosity, $\eta_{l}$, of the dust fluid. This transport coefficient $\left(\eta_{l}\right)$ is connected to the shear $\eta_{s}$ and bulk, $\eta_{b}$ viscosities via the relation $\eta_{l}=\left(4 \eta_{s} / 3\right)+\eta_{b}$. Obtaining these quantities is not a straightforward task and, in general, they must be found empirically by either experimental measurement or molecular dynamic (MD) simulations [24], in conjunction with, for example, the Green-Kubo relations [25]. Such simulations are generally based on the Debye-Hückel model and are applicable in a broad range of physical systems. A number of papers provide tabular and graphical results obtained via these methods [26-30]. For this study, we note that the bulk viscosity is typically three orders of magnitude smaller than the shear viscosity [29], and so we will only consider the shear component in this paper, such that $\eta_{l} \approx \eta_{s}$. We obtain this value of $\eta_{s}$ from the work of Sanbonmatsu and Murillo [28], who spatially modulated the velocity of the particles in a MD simulation and calculated the shear viscosity from the relaxation time of the modulation profile for a range of values of $\Gamma$ and $\kappa$.

The net force on the dust particles is thus a combination of these three forces, and this is reflected in the momentum equation,

$$
m_{d} n_{d}\left(\frac{\partial u_{d}}{\partial t}+u_{d} \frac{\partial u_{d}}{\partial x}\right)=Z_{d} e n_{d} \frac{\partial \Phi}{\partial x}-\frac{\partial P_{\star}}{\partial x}+\eta_{l} \frac{\partial^{2} u_{d}}{\partial x^{2}} .
$$

Finally, Poisson's equation gives the electrostatic potential generated from a given distribution of charges,

$$
\frac{\partial^{2} \Phi}{\partial x^{2}}=-\frac{e}{\epsilon_{0}}\left(n_{i}-n_{e}-Z_{d} n_{d}\right),
$$

where we have assumed that the ions have a charge number $Z_{i}=1$ for simplicity. Since the mass of the electrons and ions is much less than that of the dust particles, we assume that, when the dust particles are perturbed due to the passing of the wave, the electrons and ions instantaneously redistribute themselves according to the Maxwell-Boltzmann distribution, such that $n_{e}=n_{e 0} \exp \left(e \Phi / k_{B} T_{e}\right)$ and $n_{i}=n_{i 0} \exp \left(-e \Phi / k_{B} T_{i}\right)$.

We note that though we do not include ionization of neutral particles in our model, the physical reality is that electrons and ions continuously arrive at the dust particles and recombine on their surfaces, and so ionization must be taking place somewhere in the system to maintain this dynamic equilibrium. Furthermore, we note that at low gas pressures, plasma ionization becomes important for ion-acoustic and dust-acoustic waves as demonstrated by D'Angelo [31,32]. This effect is omitted here for simplicity and analytical tractability.

For notational clarity, we normalize the dynamic variables appearing in Eqs. (1), (4), and (5). The effective electrostatic temperature is scaled such that $d=T_{\star} / T_{0}$, where $T_{0}=$
$Z_{d}^{2} n_{d 0} T_{i} T_{e} /\left(n_{i 0} T_{e}+n_{e 0} T_{i}\right)$. The dust density, dust velocity, and electrostatic potential are then scaled as $n=n_{d} / n_{d 0}, u=$ $u_{d} / \sqrt{k_{B} T_{0} / m_{d}}$ and $\phi=\Phi /\left(k_{B} T_{0} / Z_{d} e\right)$, respectively. The spacelike and timelike variables are scaled by the Debye length, $\lambda_{D 0}=\sqrt{\epsilon_{0} k_{B} T_{0} / n_{d 0} Z_{d}^{2} e^{2}}$, and plasma period $\omega_{\mathrm{pd}}^{-1}=$ $\sqrt{\epsilon_{0} m_{d} / n_{d 0} Z_{d}^{2} e^{2}}$, respectively, and the normalized version of these quantities is denoted with a tilde. The longitudinal viscosity is normalized such that $\eta=\eta_{l} / m_{d} n_{d 0} \omega_{\mathrm{pd}} \lambda_{D 0}^{2}$. Equations (1), (4), and (5) thus become

$$
\begin{gathered}
\frac{\partial n}{\partial \tilde{t}}+\frac{\partial}{\partial \tilde{x}}(n u)=0 \\
n\left(\frac{\partial u}{\partial \tilde{t}}+u \frac{\partial u}{\partial \tilde{x}}\right)=n \frac{\partial \phi}{\partial \tilde{x}}-\frac{\partial(n d)}{\partial \tilde{x}}+\eta \frac{\partial^{2} u}{\partial \tilde{x}^{2}},
\end{gathered}
$$

and

$$
\frac{\partial^{2} \phi}{\partial \tilde{x}^{2}}=n+\frac{\mu}{1-\mu} \exp \left(\sigma_{e} \phi\right)-\frac{1}{1-\mu} \exp \left(-\sigma_{i} \phi\right),
$$

with $\mu=n_{e 0} / n_{i 0}, \theta=T_{i} / T_{e}, \sigma_{i}=(1-\mu) /(1+\mu \theta)$, and $\sigma_{e}=\theta \sigma_{i}$. This is a modified version of the well-known generalized hydrodynamic model [33-35], with the inclusion of the dust electrostatic pressure term accounting for strongcoupling effects.

Since we are interested in weakly nonlinear waves and there are no external electric fields applied to the system, we assume that the normalized potential is small, such that $\phi \ll 1$. As a consequence of this, we may expand the exponential functions appearing in Eq. (8) such that $\exp (j \phi)=1+j \phi+(j \phi)^{2} / 2+$ $\mathcal{O}\left(\phi^{3}\right)$. For the purpose of deriving Burgers's equation for this system, we only require up to the $\phi^{2}$ term. With that approximation, Poisson's equation simplifies to

$$
\frac{\partial^{2} \phi}{\partial \tilde{x}^{2}}=(n-1)+c_{1} \phi+c_{2} \phi^{2}+\mathcal{O}\left(\phi^{3}\right),
$$

where

$$
c_{1}=1, \quad c_{2}=-\frac{(1-\mu)\left(1-\mu \theta^{2}\right)}{2(1+\mu \theta)^{2}} .
$$

\section{REDUCTIVE PERTURBATION METHOD}

Since we are dealing with weak nonlinearities, we may linearize the equations using the reductive perturbation method, whereby we expand the dynamical quantities in terms of a small parameter $\epsilon$. We then expand the dust number density $n$, the dust fluid velocity $u$, and the electrostatic potential $\phi$ such that

$$
\begin{gathered}
n=1+\epsilon n_{1}+\epsilon^{2} n_{2}+\cdots, \\
u=\epsilon u_{1}+\epsilon^{2} u_{2}+\cdots,
\end{gathered}
$$

and

$$
\phi=\epsilon \phi_{1}+\epsilon^{2} \phi_{2}+\cdots .
$$

As mentioned in Sec. I, $T_{\star}$ is also a dynamically varying quantity, depending on both $\Phi$ and $n_{d}$. For notational clarity, we expand the normalized electrostatic temperature, $d=$ $T_{\star} / T_{0}$ separately, such that

$$
d=d_{0}+\epsilon d_{1}+\epsilon^{2} d_{2}+\cdots,
$$


with the perturbations $d_{1}$ and $d_{2}$ being

$$
d_{1}=d_{11} n_{1}+d_{12} \phi_{1}
$$

and

$$
d_{2}=d_{21} n_{2}+d_{22} \phi_{2}+d_{23} n_{1}^{2}+d_{24} n_{1} \phi_{1}+d_{25} \phi_{1}^{2} .
$$

The expressions for $d_{i j}$ are presented in the Appendix, and a derivation of these quantities is given in Ref. [18].

For the sake of rigor, it may be noted that, since the viscosity coefficient is a function of both $\kappa$ and $\Gamma$ (see, e.g., Ref. [24]), its value may in principle be perturbed by the passing shock. Therefore, one might consider $\eta \simeq \eta_{0}+$ $\epsilon \eta_{1}+\epsilon^{2} \eta_{2}+\cdots$. However, in deriving Burgers's equation in Sec. IV below, we only require orders up to $\epsilon^{3}$ for the viscosity term in the momentum equation. Given our choice of stretched coordinates, only the equilibrium term of the viscosity contributes to terms of this order, so all higher-order perturbations of $\eta$ are not required for the present study, hence, from this point forward we take $\eta \simeq \eta_{0}$.

\section{RESULTS}

\section{A. Linear}

To obtain the linear dispersion relation for this system, we expand the state variables in Eqs. (6), (7), and (9) in a power series of $\epsilon$, as described above, with terms $\epsilon^{2}$ or higher neglected. By then assuming oscillatory solutions to the perturbed quantities, such that $\partial / \partial \tilde{t} \rightarrow-i \tilde{\omega}$ and $\partial / \partial \tilde{x} \rightarrow i \tilde{k}$, we obtain the linear dispersion relation

$$
\tilde{\omega}^{2}+i \tilde{\omega} \tilde{k}^{2} \eta=\frac{\alpha \tilde{k}^{2}}{1+\tilde{k}^{2}}+\beta \tilde{k}^{2},
$$

in which we have

$$
\alpha=1-d_{12}, \quad \beta=d_{0}+d_{11} .
$$

It may be deduced from their form in the Appendix that the electrostatic temperature coefficients $d_{11}$ and $d_{12}$ are positive quantities for dusty plasmas for which both $\mu$ and $\theta$ are less than unity. For the parameters considered in this paper, these two conditions are satisfied. From this observation, we see that in this case $\alpha \leqslant 1$ and $\beta \geqslant 0$. For weakly coupled dusty plasmas (for which $\kappa_{0} \gg 1$ and $\Gamma \ll 1$ ) the electrostatic temperature terms tend to zero, in which case $\alpha=1$ and $\beta=0$.

For the purposes of the following discussion, we separate the dispersion relation into its real and imaginary components by first setting $\tilde{\omega}=\tilde{\omega}_{R}+i \tilde{\omega}_{I}$. Thus, by Eq. (17), we obtain $\tilde{\omega}_{R}^{2}-\tilde{\omega}_{I}^{2}-\tilde{\omega}_{I} \eta \tilde{k}=\alpha \tilde{k}^{2} /\left(1+\tilde{k}^{2}\right)+\beta \tilde{k}^{2}$ and $\tilde{\omega}_{R}\left(2 \tilde{\omega}_{I}+\right.$ $\left.\tilde{k}^{2} \eta\right)=0$ as the real and imaginary components, respectively. For nonzero real frequency, the imaginary component reduces to $\tilde{\omega}_{I}=-\tilde{k}^{2} \eta / 2$. This reflects the energy dissipation associated with the viscosity.

We first consider the nondissipative case $(\eta=0)$, which leads to the dispersion relation $\tilde{\omega}^{2}=\alpha \tilde{k}^{2} /\left(1+\tilde{k}^{2}\right)+\beta \tilde{k}^{2} \equiv$ $\tilde{\omega}_{0}^{2}$. From this, it is found immediately that for both weak and strong-coupling models an acoustic mode is obtained for small $\tilde{k}$, with the real frequency being proportional to $\tilde{k}$, such that $\tilde{\omega}_{R} \simeq(\alpha+\beta)^{1 / 2} \tilde{k}$. While in the strongly coupled case $\tilde{\omega}_{R}$ remains proportional to $\tilde{k}$ for large $\tilde{k}$, with $\tilde{\omega}_{R} \simeq \beta^{1 / 2} \tilde{k}$, in the weakly coupled model the frequency saturates at $\omega=\omega_{\text {pd }}$, the dust plasma frequency for the weakly coupled mode.
The introduction of finite dissipation leads to $\tilde{\omega}_{R}^{2}=\tilde{\omega}_{0}^{2}(\tilde{k})-$ $\eta^{2} \tilde{k}^{4} / 4$, and so for $\tilde{k} \ll 1$ this is seen to have only a small effect on the dispersion, which is still described by an acoustic curve of slightly lower value. However, an interesting feature of the damped case $(\eta>0)$ is that it follows directly from Eq. (16) that for $\tilde{k}$ greater than some critical value $\tilde{k}_{\mathrm{cr}}$, the real frequency $\tilde{\omega}_{R}=0$. This is given by

$$
\tilde{k}_{\mathrm{cr}}=\left[\frac{\left.-\left(\eta^{2}-4 \beta\right)+\sqrt{\left(\eta^{2}+4 \beta\right)^{2}+16 \eta^{2} \alpha}\right)}{2 \eta^{2}}\right]^{1 / 2}
$$

and applies to both the weakly and strongly coupled cases. Further, for both cases, the damping $\left(\left|\tilde{\omega}_{I}\right|\right)$ increases as $\tilde{k}^{2}$ (viz. $\tilde{\omega}_{I}=-\eta \tilde{k}^{2} / 2$ ) for $\tilde{k}<\tilde{k}_{\mathrm{cr}}$. Physically, this corresponds to a nonpropagating damped mode (further algebraic details omitted here). Note that the limit of the above expression for vanishing $\eta$ is infinity, thus the condition for the nonpropagating mode to occur $\left(\tilde{k}>\tilde{k}_{\text {crit }}\right)$ is never satisfied in this case, as expected.

As $\tilde{k}$ is increased through $\tilde{k}_{\mathrm{cr}}$, two zero-frequency, strongly damped modes appear. Both the phase and group velocities vanish for such zero-frequency modes, and no energy is transmitted. They do not appear to have any physical significance. In particular, as they occur for short wavelengths, they have no effect on the Burgers's discussion that follows, as that assumes small $\tilde{k}$. We note that the zero-frequency modes arise from the imaginary term in the dispersion relation and thus also occur in the case of other dissipation mechanisms such as dust-neutral collisions.

This behavior of the wave modes is illustrated in Fig. 1, in which we present the real and imaginary parts of the frequency as functions of wave number, both with and without the dissipative term. Figure 1(a) shows the weakly coupled case, with $\alpha=1$ and $\beta=0$, while in Fig. 1(b) we have included the effects of strong coupling. The parameters used are taken from Rosenberg et al. [20] and yield values $\alpha=0.79$ and $\beta=1.2$. Note that, contrary to Ref. [20], we do not assume that the dust particles are characterized by high kinetic temperatures of $T_{d} \sim 10-80 \mathrm{eV}$ but use a standard assumption that the dust is in thermal equilibrium with ions, $T_{d} \sim T_{i}$. The value of the viscosity was obtained by first calculating the equilibrium coupling parameter, $\Gamma$, and the normalized interparticle distance, $\kappa_{0}$, for this system. These values were then compared to Figs. 1 and 2 in Ref. [28] to obtain a value for the normalized shear viscosity. Multiplying this value by $4 / 3$, and assuming that the bulk viscosity is negligible, we then obtain the normalized longitudinal viscosity, $\eta$.

The nondissipative case is represented by the dashed blue curves in the two figures, which confirm the discussion of the undamped acoustic wave outlined above for the two models under consideration. The continuous red curves show the dispersion in the presence of viscosity. One notes that the dissipation has a minimal effect on the long-wavelength dispersion of the wave in both figures. We also see that both the strongly and weakly coupled models have dispersion curves of similar shape, with an acoustic mode for small $\tilde{k}$, and then a flattening of the curve, followed by a reduction in real frequency as $\tilde{k}$ is increased, until the zero-frequency mode is found for all $\tilde{k}>\tilde{k}_{\mathrm{cr}}$. However, it is seen that the critical wave number $\tilde{k}_{\mathrm{cr}}$ is significantly larger for the strongly coupled 

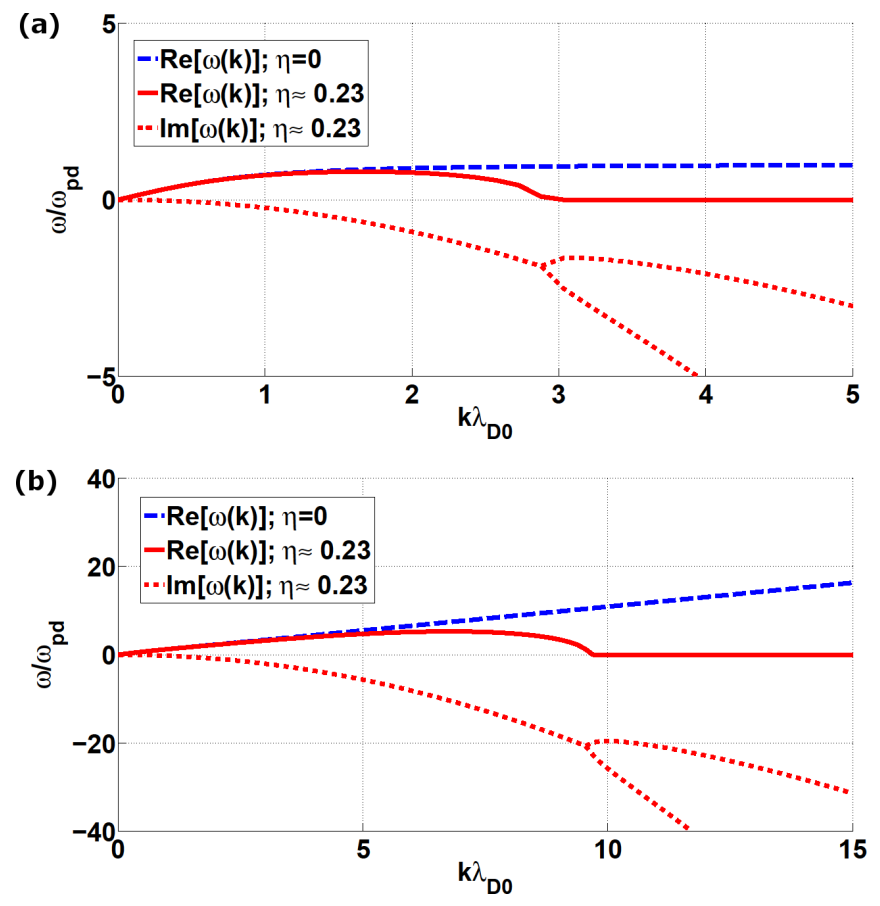

FIG. 1. (Color online) Real (positive, upper curves) and imaginary (negative, lower curves) parts of the linear dispersion relation for dust-acoustic waves in both (a) weakly and (b) strongly coupled dusty plasmas. Here we have $n_{i 0}=5 \times 10^{14} \mathrm{~m}^{-3}, n_{e 0}=3.3 \times$ $10^{14} \mathrm{~m}^{-3}, n_{d 0}=1 \times 10^{11} \mathrm{~m}^{-3}, Z_{d}=1700, k_{B} T_{i}=0.05 \mathrm{eV}$, and $k_{B} T_{e}=2.5 \mathrm{eV}$.

model than for the weakly coupled case in Fig. 1(a), that is, the finite frequency mode can be found over a wider wavelength range, although it is increasingly damped as $\tilde{k}$ is increased (dotted red curves).

\section{B. Nonlinear shocks}

To obtain Burgers's equation, we use the moving and stretched reference frame such that

$$
\tilde{\xi}=\epsilon(\tilde{x}-v \tilde{t}), \quad \tilde{\tau}=\epsilon^{2} \tilde{t},
$$

where $v$ is the linear phase velocity of the DAW or the sound speed of the plasma. Then, to lowest order, the expansion of Eqs. (6)-(9) leads to the relations

$$
\begin{aligned}
& v=\sqrt{1+d_{0}+d_{11}-d_{12}}, \\
& n_{1}=-\phi_{1}, \quad u_{1}=-v \phi_{1} .
\end{aligned}
$$

To next order, one may obtain Burgers's equation for this system such that

$$
\frac{\partial \phi_{1}}{\partial \tilde{\tau}}+\tilde{A} \phi_{1} \frac{\partial \phi_{1}}{\partial \tilde{\xi}}=\tilde{C} \frac{\partial^{2} \phi_{1}}{\partial \tilde{\xi}^{2}},
$$

in which we have

$$
\tilde{A}=-\frac{\left(1+2 v^{2}+2 \alpha c_{2}+2 \gamma\right)}{2 v}, \quad \tilde{C}=\frac{\eta}{2},
$$

where $\alpha$ is defined in Eq. (18) and $\gamma$ is defined as

$$
\gamma=d_{11}-d_{12}+d_{23}-d_{24}+d_{25} \text {. }
$$

Equation (23) admits shock solutions of the form

$$
\phi_{1}(\tilde{\xi}, \tilde{\tau})=\phi_{m}\left[1-\tanh \left(\frac{\tilde{\chi}}{\tilde{\Delta}}\right)\right]
$$

where $\tilde{\chi}=\tilde{\xi}-\tilde{U} \tilde{\tau}, \phi_{m}=\tilde{U} / \tilde{A}$ is the amplitude of the shock, $\tilde{\Delta}=2 \tilde{C} / \tilde{U}$ is the normalized width, and $\tilde{U}$ is the normalized velocity of the nonlinear wave in the moving reference frame.

Given the form of Eq. (26), it is clear that both the amplitudes and widths of the shocks are dependent on $\tilde{U}$, the speed of the shock relative to the sound speed. In particular, in any specified plasma system, faster shocks will have larger amplitudes and narrower widths than slower shocks. Also, it is interesting to note that the quantity $\phi_{m} \tilde{\Delta}$ is independent of the speed of the shock in any particular system.

In a laboratory situation, one is likely to measure both the sound speed and the shock speed directly. However, from a theoretical perspective, an underestimation of the sound speed in a dusty plasma may occur if one does not account for the effects of strong coupling in the model. For example, for the normalization we have chosen, the sound speed in a weakly coupled dusty plasma system, that is, without including the electrostatic temperature terms, is scaled to unity. Using the typical plasma parameters displayed in Table I in Sec. V (System A), the sound speed in our strongly coupled model is predicted to be $v \approx 1.41$. This then has to be taken into account when choosing the value of $\tilde{U}$ for the shocks. Furthermore, since the lower bound for the speed of shock waves in the laboratory frame is $v$, this model predicts that shocks do not occur with a laboratory speed $V$ such that $1 \leqslant V \leqslant v$, which are predicted in a weakly coupled plasma.

In addition to $\tilde{U}$, the amplitude of a shock wave is dependent on the $\tilde{A}$ coefficient in Burgers's equation. In this paper, we take a dynamical form of the electrostatic temperature, and by using this we obtain the value of $\tilde{A}$ as defined in Eq. (24). This is the same coefficient that was found in Ref. [18], in which solitons were studied. If we instead had neglected the perturbations and assumed $T_{\star}=T_{\star 0}$, we would obtain

$$
\tilde{A}=-\frac{3+2 c_{2}+2 d_{0}}{2 \sqrt{1+d_{0}}},
$$

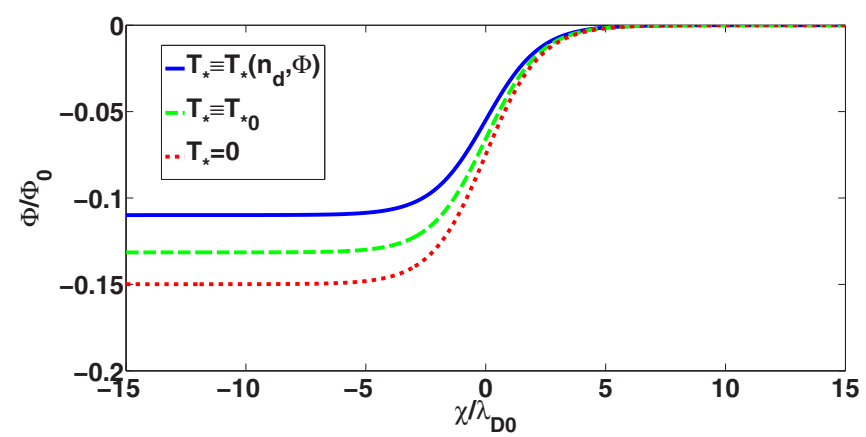

FIG. 2. (Color online) Shock solution to Burgers's equation for the three different models. Here we have $n_{i 0}=5 \times 10^{14} \mathrm{~m}^{-3}, n_{e 0}=$ $3.3 \times 10^{14} \mathrm{~m}^{-3}, n_{d 0}=1 \times 10^{11} \mathrm{~m}^{-3}, Z_{d}=1700, k_{B} T_{i}=0.05 \mathrm{eV}$, $k_{B} T_{e}=2.5 \mathrm{eV}, \eta \approx 0.23$, and $\tilde{U}=0.1$. 


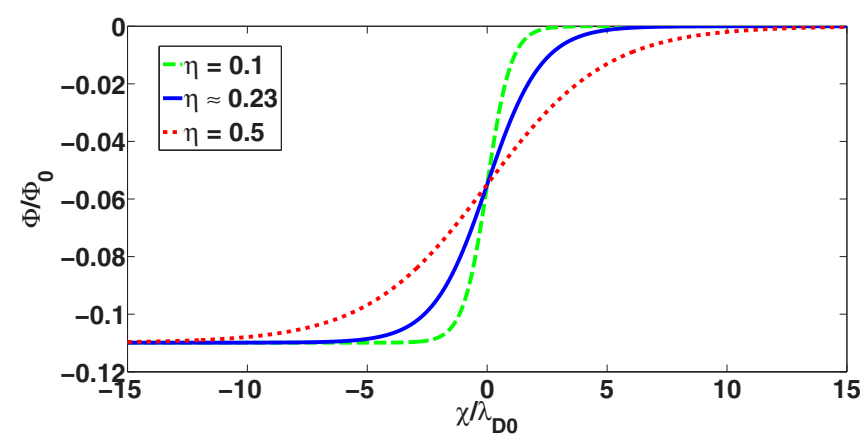

FIG. 3. (Color online) Shock solution to Burgers's equation for different dissipation coefficients. Here we have $n_{i 0}=5 \times 10^{14} \mathrm{~m}^{-3}$, $n_{e 0}=3.3 \times 10^{14} \mathrm{~m}^{-3}, n_{d 0}=1 \times 10^{11} \mathrm{~m}^{-3}, Z_{d}=1700, k_{B} T_{i}=$ $0.05 \mathrm{eV}, k_{B} T_{e}=2.5 \mathrm{eV}$, and $\tilde{U}=0.1$.

and if we neglect the electrostatic temperature terms completely, we obtain

$$
\tilde{A}=-\frac{3+2 c_{2}}{2} .
$$

For the typical dusty plasma parameters we have used, there is a significant difference in the amplitude of the shocks between the three models, as shown in Fig. 2.

The normalized widths of the shocks are only dependent on the normalized viscosity coefficient $\eta$, such that higher viscocities lead to wider shocks, which is consistent across all three models. Shock solutions for typical plasma parameters and various values for $\eta$ are shown in Fig. 3. This suggests that measuring the thickness of shocks in a plasma may be a possible method of determining the viscosity of the plasma.

\section{NUMERICAL INVESTIGATION}

In this section, we present a simple numerical investigation of the evolution of dust-acoustic shocks propagating in plasma

TABLE I. Parameters for the two regions in this numerical study.

\begin{tabular}{lcc}
\hline \hline Parameter & Region A & Region B \\
\hline Plasma parameters & & \\
$Z_{d}$ & 1700 & 1400 \\
$m_{d}(\mathrm{~kg})$ & $1 \times 10^{-15}$ & $1 \times 10^{-15}$ \\
$n_{d 0}\left(\mathrm{~m}^{-3}\right)$ & $1 \times 10^{11}$ & $1.2 \times 10^{11}$ \\
$n_{i 0}\left(\mathrm{~m}^{-3}\right)$ & $5 \times 10^{14}$ & $2.625 \times 10^{14}$ \\
$n_{e 0}\left(\mathrm{~m}^{-3}\right)$ & $3.3 \times 10^{14}$ & $9.45 \times 10^{13}$ \\
$k_{B} T_{i}(\mathrm{eV})$ & 0.05 & 0.05 \\
$k_{B} T_{e}(\mathrm{eV})$ & 2.5 & 2.5 \\
Viscosity calculations & & \\
$\Gamma$ & 390 & 280 \\
$\kappa_{0}$ & 2.92 & 1.98 \\
$\eta$ & 0.23 & 0.31 \\
Burgers's equation coefficients & \\
$A$ & -1.82 & -1.51 \\
$C$ & 0.11 & 0.16 \\
Initial conditions & & \\
$\tilde{U}$ & 0.1 & 0.1 \\
$\phi_{m}$ & -0.055 & -0.066 \\
$\Delta$ & 2.27 & 3.15 \\
\hline \hline
\end{tabular}

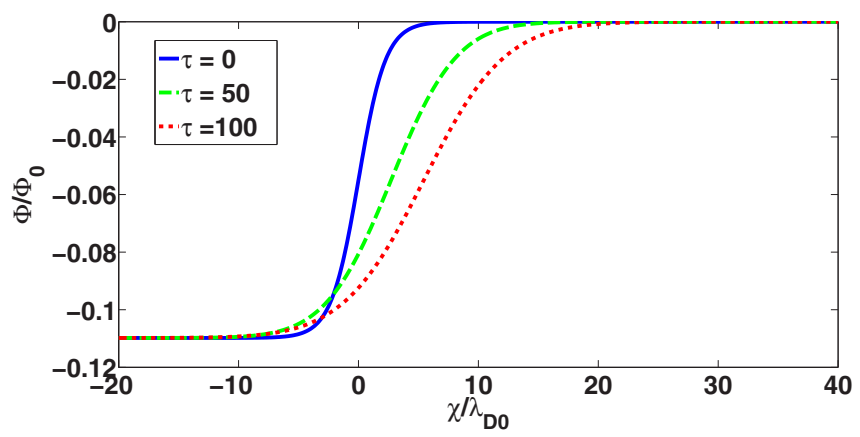

FIG. 4. (Color online) Shock originating in Region A, propagating in Region B.

conditions that are different to those in which they formed. Physically, these changing conditions may occur as the shock propagates along the discharge chamber, for example.

Again, we use the parameters presented by Rosenberg et al. [20], but we also have an additional region of higher dust density. For notational convenience, we denote the set of parameters from Rosenberg et al. [20] Region A and the region of greater dust density Region B. We then use a Runge-Kutta method to numerically integrate Burgers's equation for Region B using the shock solution of Region A as an initial condition and vice versa. The parameters for these two regions, as well as the initial conditions used in the simulations, are presented in Table I. The normalized longitudinal viscosity, $\eta$, was determined for each system using the method described in Sec. IV A. The various scaling parameters, $\lambda_{D 0}$ and $\omega_{\mathrm{pd}}$, for example, differ for each region since the plasma parameters
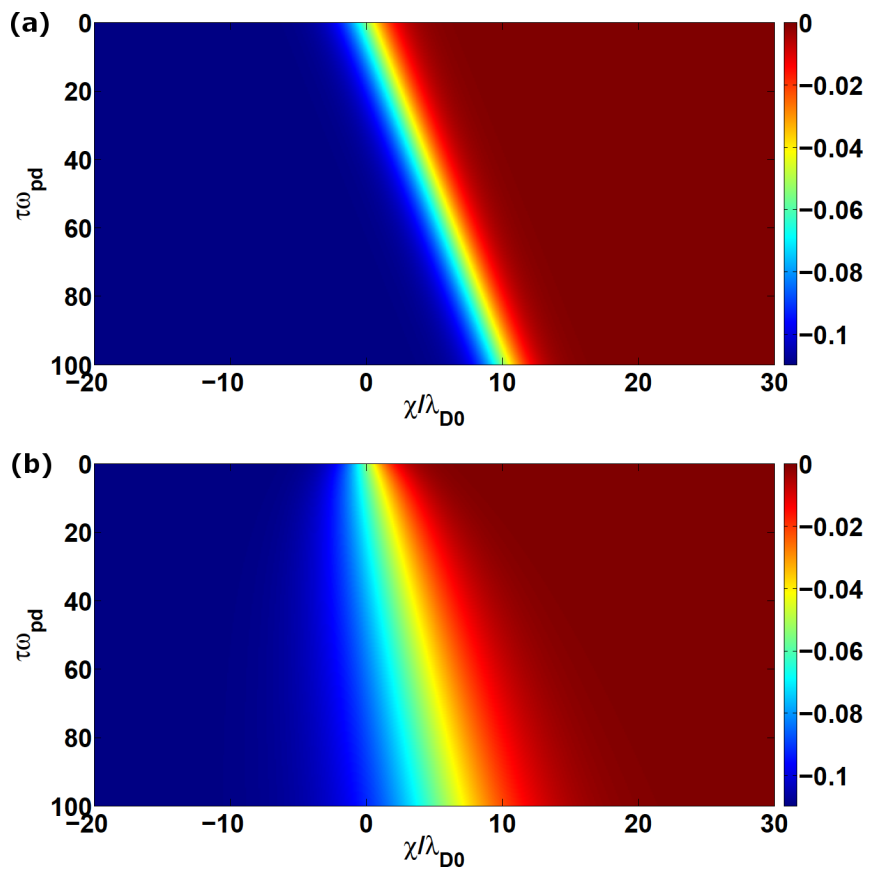

FIG. 5. (Color online) Contour plots of a shock which is a solution to Burgers's equation for Region A. Plot (a) shows that the shock is stable in Region A, whereas plot (b) shows that if this shock is propagating in Region B its width increases with time. 


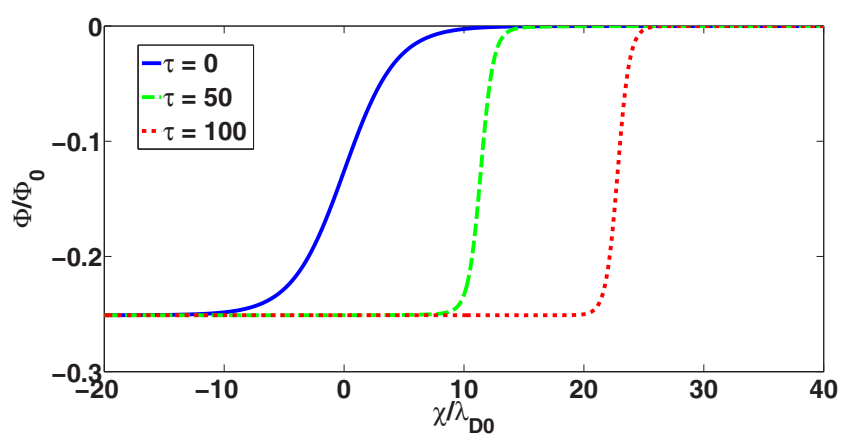

FIG. 6. (Color online) Shock originating in Region B, propagating in Region A.

have been modified. To directly compare the shocks arising from these two sets of parameters, it is more convenient to first convert them to the same normalizations. For the simulations in this section, we have converted the relevent parameters for Region $\mathrm{B}$ into the scaling of Region $\mathrm{A}$, such that the shock parameters for Region $\mathrm{B}$ become $\phi_{m} \approx-0.13$ and $\Delta \approx 4.35$, while its Burgers's coefficients become $\tilde{A} \approx-1.00$ and $\tilde{C} \approx 0.27$.

First, we consider the propagation of a dust-acoustic shock that is initially a solution to the Burgers's equation of Region A, propagating in Region $\mathrm{B}$. The increase in the number density of dust particles in the plasma for Region B has the effect of reducing $\kappa_{0}$ due to both a decrease in the interparticle spacing and an increase in the screening length since both the electron and ion densities are reduced. For the calculated value of $\Gamma$ for each region, this reduction of $\kappa_{0}$ results in a significant increase in the viscosity of the plasma and thus also an increase in the width of the shock solutions.

Figure 4 shows the result of this numerical experiment, showing an increase in the width of the shock over time. The speed of the shock is also reduced, which may be seen by a comparison of Figs. 5(a) and 5(b).

Conversely, a shock that is a solution to Region B propagating in Region A is found to experience a reduction in its width over time, as illustrated in Fig. 6. Furthermore, the speed of the shock increases when it propagates in Region A. This is shown in Figs. 7(a) and 7(b).

\section{CONCLUSION}

To summarize, we have theoretically investigated dustacoustic shocks in a dusty plasma using the phenomenological approach of Gozadinos et al. [10], which was originally developed for crystalline plasmas but has since been applied successfully in the study of strongly coupled plasmas near the liquid-crystal phase transition. For analytical tractability, we have assumed weak-amplitude excitations and proceeded by adopting a perturbative technique. Although technically limited to small amplitudes, the qualitative results obtained in our investigation can be extended to larger amplitudes (e.g., as initial conditions for numerical investigations) and may thus provide insight on the salient features of shock structures in strongly coupled dusty plasmas. Based on the linear dispersion relation and the weakly nonlinear Burgers's equation, which includes dissipative effects associated with viscosity, for the
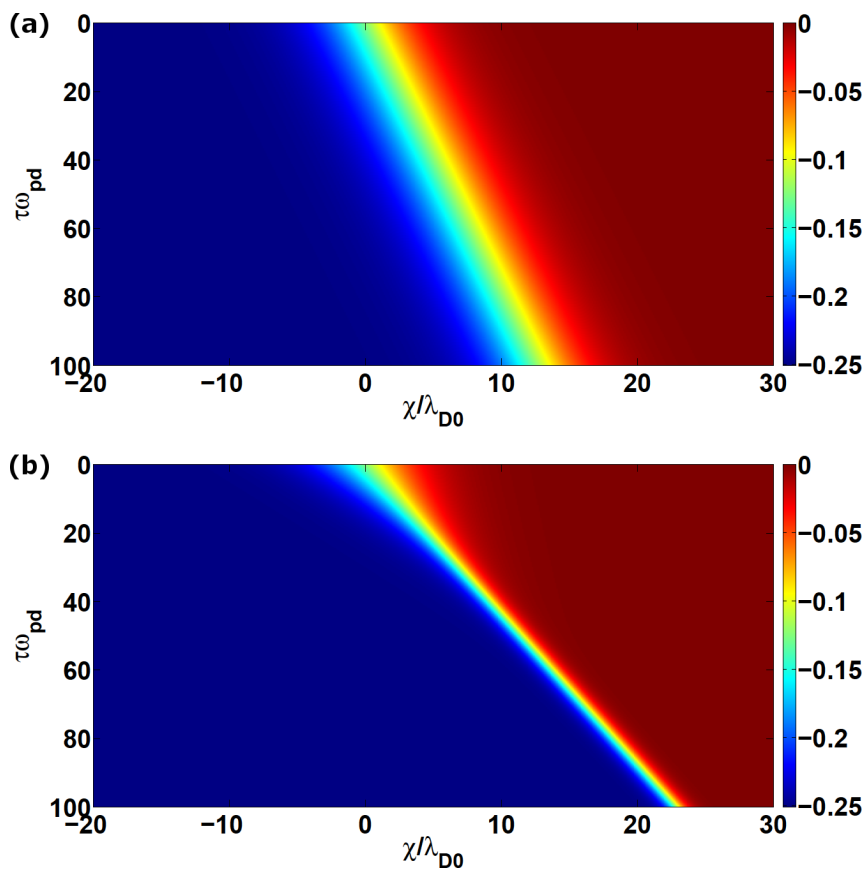

FIG. 7. (Color online) Contour plots of a shock which is a solution to Burgers's equation for Region B. Plot (a) shows that the shock is stable in Region B, whereas plot (b) shows that if this shock is propagating in Region A its width decreases with time.

typical parameter values that we have used, the results from this model suggest the following, in comparison to the weakly coupled model:

Linear dust-acoustic waves:

(i) Strong coupling mitigates the effects of dissipation in the dusty plasma such that larger wave numbers and higherfrequency waves may exist.

(ii) In the long-wavelength limit, strong coupling leads to an increase of the sound speed in the plasma.

Weakly nonlinear dust-acoustic shocks:

(i) The increase in the sound speed due to strong coupling implies a larger minimum velocity for observable dustacoustic shocks.

(ii) For a specified shock speed in the laboratory frame, the sound speed excess, $\tilde{U}$, is reduced due to the increase in predicted sound speed.

(iii) For a specified sound speed excess, $\tilde{U}$, strong coupling reduces the amplitude of the predicted shocks due to an increase in the magnitude of the nonlinearity coefficient, $\tilde{A}$.

(iv) In this model, the width of the shocks is not altered by strong-coupling effects. The width of the shocks is, however, directly proportional to the viscosity coefficient, $\eta$. This suggests that measurements of the shock thickness, together with the simulations, may be employed to obtain more accurate values of the viscosity of a dusty plasma.

Note added in proof. Recently, we were alerted to a related article, namely, M. Shahmansouri and M. Rezaei [36]. Although the article is based on a similar model to ours, we note that the similarity is limited to the algebraic structures. Neither the way that article proceeds, nor the actual research outcomes, overlap our work here. In addition, we note that they have apparently used an alternative definition of the viscosity 
coefficient $\eta$, which has different dimensions to those of the viscosity used here and elsewhere, e.g., Refs. [15,33-35].

\section{ACKNOWLEDGMENTS}

M.A.H. thanks the National Research Foundation of South Africa for partial support under Grant No. UID 85408, and acknowledges that opinions, findings, and conclusions or recommendations expressed in this publication are those of the authors, and that the NRF accepts no liability whatsoever in this regard.

\section{APPENDIX: ELECTROSTATIC TEMPERATURE COEFFICIENTS}

We take the expansion of the normalized electrostatic temperature up to second order, such that

$$
d=d_{0}+\epsilon d_{1}+\epsilon^{2} d_{2},
$$

where the equilibrium electrostatic temperature, in normalized units, is given as

$$
d_{0}=T_{\star 0} / T_{0},
$$

with

$$
k_{B} T_{\star 0}=\frac{N_{n n} Z_{d}^{2} e^{2}}{12 \pi \epsilon_{0}} \sqrt[3]{n_{d 0}}\left(1+\kappa_{0}\right) \exp \left(-\kappa_{0}\right),
$$

where $\kappa_{0}=1 / \sqrt[3]{n_{d 0}} \lambda_{D 0}$
The perturbations $d_{1}$ and $d_{2}$ are

$$
d_{1}=d_{11} n_{1}+d_{12} \phi_{1}
$$

and

$$
\begin{gathered}
d_{2}=d_{21} n_{2}+d_{22} \phi_{2}+d_{23} n_{1}^{2}+d_{24} n_{1} \phi_{1}+d_{25} \phi_{1}^{2}, \\
d_{11}=d_{21}=\frac{d_{0}}{3} \frac{1+\kappa_{0}+\kappa_{0}^{2}}{1+\kappa_{0}}, \\
d_{12}=d_{22}=-d_{0} c_{2} \frac{\kappa_{0}^{2}}{1+\kappa_{0}}, \\
d_{23}=\frac{d_{0}}{18} \frac{\kappa_{0}^{3}-3 \kappa_{0}^{2}-2 \kappa_{0}-2}{1+\kappa_{0}}, \\
d_{24}=-\frac{d_{0}}{3} c_{2} \frac{\kappa_{0}^{2}\left(\kappa_{0}-1\right)}{\left(1+\kappa_{0}\right)}, \\
d_{25}=-\frac{d_{0}}{2}\left(3 c_{3}-c_{2}^{2} \kappa_{0}\right) \frac{\kappa_{0}^{2}}{1+\kappa_{0}},
\end{gathered}
$$

where $c_{2}$ is defined by Eq. (10), and

$$
c_{3}=\frac{(1-\mu)^{2}\left(1+\mu \theta^{3}\right)}{6(1+\mu \theta)^{3}} .
$$

[1] R. Z. Sagdeev, Reviews of Plasma Physics, Vol. 4, edited by M. A. Leontovich (Consultants Bureau, New York, 1966), p. 23.

[2] H. Washimi and T. Taniuti, Phys. Rev. Lett. 17, 996 (1966).

[3] P. K. Shukla and A. A. Mamun, Introduction to Dusty Plasma Physics (Institute of Physics, Bristol, 2002).

[4] F. Verheest, Waves in Dusty Space Plasmas (Kluwer Academic, Dordrecht, 2000).

[5] R. L. Merlino, Plasma Physics Applied, edited by C. Grabbe (Transworld Research, Kerala, 2006), p. 74.

[6] N. N. Rao, P. K. Shukla, and M. Y. Yu, Planet. Space Sci. 38, 543 (1990).

[7] P. K. Shukla and V. P. Silin, Phys. Scripta 45, 508 (1992).

[8] A. Barkan, R. L. Merlino, and N. D’Angelo, Phys. Plasmas 2 , 3563 (1995).

[9] H. Ikezi, Phys. Fluids 29, 1764 (1986).

[10] G. Gozadinos, A. V. Ivlev, and J. P. Boeuf, New J. Phys. 5, 32 (2003).

[11] V. V. Yaroshenko, F. Verheest, H. M. Thomas, and G. E. Morfill, New J. Phys. 11, 073013 (2009).

[12] V. V. Yaroshenko, V. Nosenko, M. A. Hellberg, F. Verheest, H. M. Thomas, and G. E. Morfill, New J. Phys. 12, 073038 (2010).

[13] V. V. Yaroshenko, M. H. Thoma, H. M. Thomas, and G. E. Morfill, IEEE Trans. Plasma Sci. 38, 869 (2010).
[14] V. V. Yaroshenko, V. Nosenko, and G. E. Morfill, Phys. Plasmas 17, 103709 (2010).

[15] A. A. Mamun and P. K. Shukla, Europhys. Lett. 87, 55001 (2009).

[16] A. A. Mamun, K. S. Ashrafi, and P. K. Shukla, Phys. Rev. E 82, 026405 (2010).

[17] V. Nosenko, S. K. Zhdanov, S.-H. Kim, J. Heinrich, R. L. Merlino, and G. E. Morfill, Europhys. Lett. 88, 65001 (2009).

[18] S. E. Cousens, S. Sultana, I. Kourakis, V. V. Yaroshenko, F. Verheest, and M. A. Hellberg, Phys. Rev. E 86, 066404 (2012).

[19] S. Khrapak, D. Samsonov, G. Morfill, H. Thomas, V. Yaroshenko, H. Rothermel, T. Hagl, V. Fortov, A. Nefedov, V. Molotkov, O. Petrov, A. Lipaev, A. Ivanov, and Y. Baturin, Phys. Plasmas 10, 1 (2003).

[20] M. Rosenberg, E. Thomas, Jr., and R. L. Merlino, Phys. Plasmas 15, 073701 (2008)

[21] P. K. Shukla, Phys. Plasmas 8, 1791 (2001).

[22] V. E. Fortov, A. V. Ivlev, S. A. Khrapak, A. G. Khrapak, and G. E. Morfill, Phys. Rep. 421, 1 (2005).

[23] U. Konopka, G. E. Morfill, and L. Ratke, Phys. Rev. Lett. 84, 891 (2000).

[24] A. Budea, A. Derzsi, P. Hartmann, and Z. Donko, Contrib. Plasma Phys. 52, 194 (2012).

[25] J. P. Hansen and I. McDonald, Theory of Simple Liquids (Academic Press, New York, 1986). 
[26] S. Ichimaru and S. Tanaka, Phys. Rev. Lett. 56, 2815 (1986).

[27] M. S. Murillo, Phys. Rev. E 62, 4115 (2000).

[28] K. Y. Sanbonmatsu and M. S. Murillo, Phys. Rev. Lett. 86, 1215 (2001).

[29] G. Salin and J.-M. Caillol, Phys. Rev. Lett. 88, 065002 (2002).

[30] T. Saigo and S. Hamaguchi, Phys. Plasmas 9, 1210 (2002).

[31] N. D’Angelo, Phys. Plasmas 4, 3422 (1997).
[32] N. D’Angelo, Phys. Plasmas 5, 3155 (1998).

[33] P. K. Kaw and A. Sen, Phys. Plasmas 5, 3552 (1998).

[34] A. A. Mamun, P. K. Shukla, and T. Farid, Phys. Plasmas 7, 2329 (2000).

[35] P. K. Shukla and A. A. Mamun, IEEE Trans. Plasma Sci. 29, 221 (2001).

[36] M. Shahmansouri and M. Rezaei, Astrophys. Space Sci. 351, 197 (2014). 